\title{
BIM 技术在水利工程中的应用
}

\section{Application of BIM Technology in Water Conservancy Projects}

\section{辛路}

Lu Xin

中国水利水电第六工程局有限公司

中国·辽宁 沈阳 110179

China Water and HydropowerSixth Engineering Bureau Co.,Ltd.,

Shenyang, Liaoning, 110179, China
【摘要】在水利工程建设过程中应用 BIM 技术, 不仅可以使水利工程建设的查询可视化, 更加严格准确地控制关键点的质量，还可以有效地将工程管理方面的技术和信息方面的 技术进行融合,从而使水利工程信息化建设得到前所未有的提高。

【Abstract】The application of BIM technology in water conservancy engineering construction process, not only can make query visualization of construction of water conservancy projects, more strictly control the quality of the key points accurately, can also effectively will project management technology and information fusion technology, thus make the informatization construction of water conservancy project to improve on an unprecedented scale.

【关键词】水利工程;信息化; 应用

[Keywords]water conservancy project; informatization; application

【DOI】10.36012/etr.v2i1.975

\section{1 引言}

水利工程的建设关乎民生,而水利工程建设的质量直接 关乎着水利工程后续作用的发挥。水利工程因为其建设涉及 的范围较广、地形需求较为复杂, 所以在水利工程建设过程 中会存在诸多的问题,例如,信息图纸繁多、土方量计算偏差 大、工程枢纽设计杂乱等一系列问题。为了解决这些问题,专 业人员研究出构建水利工程信息模型的办法,通过构建 BIM 模型, 充分实现了水利工程的真实数据查询, 可运用众多 BIM 设计软件共同进行水利工程设计枢纽的布置及土方量 的运算等。

\section{2 水利工程建设中应用 BIM 技术的优势}

BIM 技术是一种全新的有关建筑构图的软件 [1], 在中国最 早是应用于建筑领域,软件自带 3D 视图效果, 可以进行构建 建筑模型来实现建筑效果的清晰化和具体化。在有具体模型 的主观条件之下, 相关的工作人员可以更加关注细节建设,有 效避免水利工程建设中的各种安全隐患，从而实现实际运用 与建筑设计的完美融合。

水利工程建设过程中运用 BIM 技术的优势如下: 第一, 在进行水利工程建设之前, 运用 BIM 相关技术可以较为详细
地了解水利工程建设的大体方案, 从而保证水利工程建设项 目的顺利进行。第二,在水利工程建设的过程当中,运用 BIM 相关的技术, 可以使每个部门通过 BIM 软件平台无障碍地沟 通, 在发布一些修改设计及变更的时候, 通过 BIM 交流平台 可以及时准确地使各个部门接受相关信息，从而避免信息传 达不及时而带来的危害，使水利工程建设的施工质量得到有 效的保证。第三，在相关工作人员对水利工程进行设计的时 候, 运用 BIM 技术可以较为直观地模拟出设计图纸所设计的 具体模型, 可以使设计的不足在模型中展现出来, 相关的设计 师便可根据相关问题进行图纸的修改设计，从而缩短施工工 期,使工程成本的投人得到有效的控制。总而言之,将 BIM 技 术应用于水利工程建设的过程当中，不仅可以使水工程建设 的投入成本得到有效的降低,使社会经济效益最大化,而且可 以保证水利工程的建设质量。

\section{3 水利工程建设中 BIM 技术的具体应用}

\section{1 土方量计算中 BIM 技术的应用}

土方量的测量与计算是水利工程建设中每个环节的施工 依据, 土方量计算是否准确无误,直接关乎水利工程中各个施 工环节的施工质量 ${ }^{[2]}$ 。在正常情况下,水利工程地形杂乱无章, 土方量的挖填需求比较大, 从而使在对土方量进行测量的时 
水利水电工程 Water Resources and Hydropower Engineering

候计算误差较大。而在利用 BIM 技术对土方量进行计算的时 候，现场施工场地的 3D 视图可以直接地呈现在工作人员的 面前,工作人员可以利用相关 BIM 技术对土方量进行严格的 测量, 从而使土方量测量数值的准确性及测量效率得到有效 的提高。Auto CAD 和 GIS 等有关软件都是计算土方量常用的 测量软件。

\section{2 水利枢纽建设中 BIM 技术的应用}

水利工程枢纽设置在水利工程建设过程中起着至关重要 的作用, 水利工程枢纽设置的主要目的是准确无误地确定建 筑物在平面上和高程上的相关布置。对工程建设的整体布局 起关键作用的便是枢纽的布置，水利工程建筑物体与枢纽二 者的位置准确无误地确定, 不仅有利于水利工程整体的设计, 而且对设备的运输及临时设计的决定都有非常重要的意义。 在其中运用 BIM 技术, 可以使建筑物的布置直接呈现于决策 者的面前，使设计人员可以直观地看到地形与建筑物之间的 关系。另外, BIM 技术中的 BIM 模型可以直接任意地调动, 有 关枢纽布置的工作人员可以较为科学地设置枢纽位置。

\section{$4 \mathrm{BIM}$ 技术在水利工程中的应用实例}

涵闸水利工程建设项目便是综合运用 BIM 技术来实现
的。在建设过程当中利用 BIM 技术进行水利工程建筑交通 桥、工作桥、 $U$ 型槽、底板、闸墩等一系列水利工程建筑的建模 工作, 然后通过相关 BIM 软件对水利工程建筑进行简单的组 装, 在相关 BIM 软件中可以迅速地完成一座简易的涵闸的模 型建设, 从而通过模型建设而准确获取相关的信息, 实现了准 确、迅速获取相关数据信息的目的。

\section{5 结语}

水利工程设计的过程当中, 建筑物的设计相当麻烦, 因为 设计技术较为复杂, 选型较为独特, 所以导致设计的效率普遍 偏低, 而且在水利工程建设的过程当中信息的流畅性并不是 很好, 从而限制了水利工程的施工质量及施工效率。在水利工 程建设的过程当中运用 BIM 技术可以有效地降低土方量计 算的误差, 使水利枢纽的布置具有科学性, 并且降低了水利工 程建设的投入成本, 从而使水利工程建设更加接近于信息化。

\section{参考文献}

[1]葛文兰,于晓明,何波.BIM 第二维度一一项目不同参与方的 BIM 应用[M].北京:中国建筑工业出版社,2018.

[2]苗倩. BIM 技术在水利水电工程可视化仿真中的应用 [J].水电 能源科学,2018(10):139-142. 